\title{
Numerical evaluation of fractional differ-integrals of some periodical functions via the IMT transformation
}

\author{
D. BRZEZIŃSKI* and P. OSTALCZYK \\ Faculty of Electrical, Electronic, Computer and Control Engineering, Computer Engineering Institute, Lodz University of Technology, \\ 18/22 Stefanowskiego St., 90-537 Łódź, Poland
}

\begin{abstract}
This paper presents accuracy evaluation of the numerical calculations of the fractional differ-integrals. We focus on applying the Riemann-Liouville formula, on singularity, which appears while using classical form of this formula. To calculate it we use the Newton-Cotes' Quadrature and additionally two Gaussian rules. Using this different approach to the IMT Transformation, transforming the "core" integrand of Riemann-Liouville formula, we point the possible way of increasing the accuracy of the calculations. We use our own tools and compare obtained results with, where possible, exact values, where not - values obtained using an excellent method of integration incorporated in Mathematica.
\end{abstract}

Key words: fractional-order derivatives and integrals, accuracy of numerical calculations, numerical integration, IMT Transformation, periodical functions.

\section{Introduction}

Fractional calculus has been playing recently a major role in many scientific areas. The fractional-order derivative (FOD) and integral (FOI) are natural extension of the well-known derivative and integral. This extension enables better physical phenomena identification [1,2] analysis [3] and control [4$6]$. But there are still problems in numerical evaluation of the fractional-order derivatives and integrals $[7,8]$

In this paper, several numerical methods applied to FOD/FOI calculation due to its accuracy are compared. Appropriate conclusions and remarks are derived.

The paper is organised as follows: firstly basic definitions of the Riemann-Liouville and the Grünald-Letnikov FOD and FOI and, in next section numerical methods used in calculation of the integrals are given. Then functions subjected to the fractional differentiation and integration are presented. After those the main results are gathered. Finally, the conclusions are presented.

\section{Mathematical preliminaries}

There are several formulas, which can be applied to calculate differ-integrals numerically: the Riemann-Liouville formula and the Grünwald-Letnikov method [9, 10]. The latter often used, because of its simplicity, in technical applications. They distinct from each other in one main way: the GrünwaldLetnikov formula derives from differential quotient and the Riemann-Liouville from multiple integrals.

In this paper we focus on applying the Riemann-Liouville formula and on the idea how to increase the accuracy when calculating it numerically, using some methods of numerical integration as well.
The Grünwald-Letnikov formula is used for comparing purposes only. The level of absolute errors obtained in calculations by this formula is treated as a point of reference in the context of the accuracy of the calculations.

\section{The Riemann-Liouville formula of the fractional order differ-integral (RL)}

The definite RL integral of the real function $f(t)$ of the $\nu>0$ order is defined as follows:

$$
{ }_{t_{0}} I_{t}^{\nu} f(t)=\frac{1}{\Gamma(\nu)} \int_{t_{0}}^{t}(t-\tau)^{\nu-1} f(\tau) d \tau
$$

where $t_{0}, t$ denotes integration range, which comply with the condition $-\infty<t_{0}<t<\infty, \Gamma(\nu)$ is Euler's Gamma function.

Now, we describe natural number $n$ :

$$
n=\lceil\nu\rceil+1
$$

where $n$ also denotes the order of classical derivative.

The RL derivative of the real function $f(t)$ of the $\nu>0$ order is defined as follows:

$$
\begin{gathered}
t_{0} D_{t}^{\nu} f(t)=\sum_{i=0}^{n-1} \frac{\left(t-t_{0}\right)^{i-\nu} f^{(i)}\left(t_{0}\right)}{\Gamma(i+1-\nu)} \\
+\frac{1}{\Gamma(n-\nu)} \int_{t_{0}}^{t}(t-\tau)^{n-\nu-1} f^{(n)}(\tau) d \tau .
\end{gathered}
$$

\footnotetext{
*e-mail: dbrzezinski@kis.p.lodz.pl
} 


\section{The Grünwald-Letnikov formula of the fractional order differ-integral (GrLet)}

The derivative of a real order $\nu>0$ (for the integral we use the order $-\nu<0$ ) of a continuous bounded function $f(t)$ is defined as follows

$$
t_{0} D_{t}^{\nu} f(t)=\lim _{\substack{h \rightarrow 0 \\ t-t_{0}=k h}} \frac{\sum_{i=0}^{\frac{t-t_{0}}{h}} a_{i}^{(\nu)} f(t-h i)}{h^{\nu}},
$$

where

$$
a_{i}^{(\nu)}=\left\{\begin{array}{l}
1 \quad \text { for } i=0 \\
a_{i-1}^{(\nu)}\left(1-\frac{1+\nu}{i}\right)
\end{array} \quad \text { for } \quad i=1,2,3, \ldots\right.
$$

\section{Short review of fundamentals of numerical integration. Tested functions}

In the process of calculating the differ-integrals it is necessary to calculate a value of the definite integral over the range $\left[t_{0}, t\right]$. Usually it is interpolated with the following formula

$$
\int_{t_{0}}^{t} f(t) d t=\sum_{k=0}^{L} A_{k} f\left(t_{k}\right)+R .
$$

The right side of the equation is called quadrature in which $t_{k}$ denotes quadrature nodes, $A_{k}$ quadrature coefficients (weights), $L$ number of intervals in interpolation and $R$ remainder.

The above formula is shared by all quadratures. The difference lies in the algorithms of calculating their nodes and coefficients [11-13, 15].

In table 1 specification of applied methods of numerical integration are collected.

The Special modification of the Gauss Quadrature called Gauss-Kronrod Quadrature (GaKRO) was used in a form of tabulated values of the nodes and the weights.

The method is based on Gauss-Legendre Rule. There is no need to overlap all the details about it. Suffice it to say that the G7/K15, so called Gauss-Kronrod Pair (we apply it along with G30/K61 one), includes the nodes of the 7-point Gauss-Legendre Quadrature +8 new ones and all 15 new coefficients [14]

We used the following formulas to calculate differintegrals:

- the Riemann-Liouville differ-integral (RL),

- Modified Riemann-Liouville differ-integral via mentioned at the beginning - the IMT Transformation (mRL).

Additionally we use the Grünwald-Letnikov differ-integral formula (GrLET) for comparing purposes.

Our C++ programs which were developed especially for the purpose of this experiment used following methods of numerical integration while applying formulas (RL, mRL), are:

- Newton-Cotes Quadrature, Midpoint Rule (NCM),

- Gauss-Legendre Quadrature (GaLEG),

- Gauss-Kronrod Quadrature (GaKRO).
Table 1

Important parameters used in integration rules

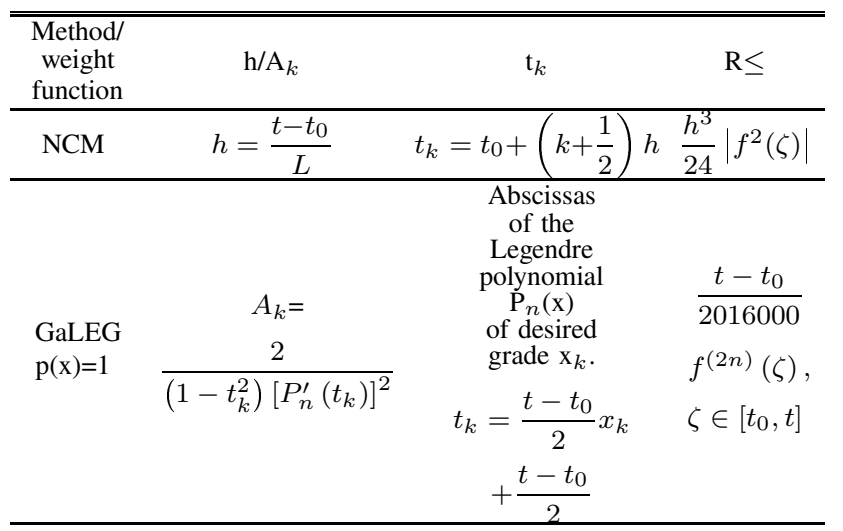

We selected two periodical functions. A graph of the second one (8) is presented in Fig. 1.

$$
\begin{gathered}
f(t)=\sin (t), t \in(0 ; 2 \pi), \\
f(t)=1.5 \cos (2 t)+2.2 \cos (4 t), t \in(0 ; 2 \pi) .
\end{gathered}
$$

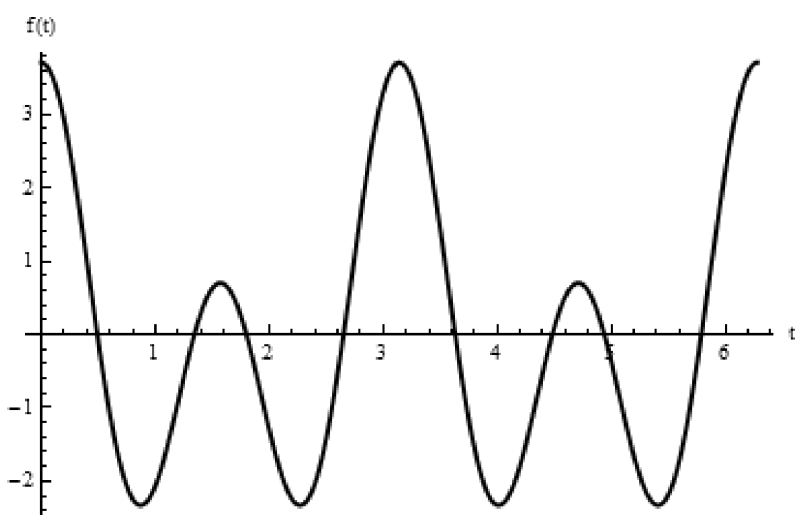

Fig. 1. Graph of the function (8)

For functions (7), (8) we calculate two types of expressions: the fractional derivative ${ }_{t_{0}} D_{t}^{\nu} f(t)$ and fractional inte$\operatorname{gral}_{t_{0}} I_{t}^{\nu} f(t)$.

Our goal was to determine how the methods will perform when using the smallest, arbitral chosen, number of sample points - possibly less than 600 (the highest, default value for GrLET in our practical applications) and which one will deliver the results burdened with the smallest absolute error.

The amount of sample points should be exactly determinable. That is why there was not any advanced technique of the integration as for example adaptive strategies applied.

The criterion of the calculation accuracy was the absolute error. That is why, in our opinion, there is no need to make such test complicated by incorporating extensive error estimation of every method applied. Additionally, theoretically estimation error formulas relate to a value of the derivative of the appropriate orders (for Newton-Cotes' Midpoint Rule $-2^{\text {nd }}$ order derivative and Gauss-Legendre Quadrature $-2 \mathrm{n}^{n d}$ order derivative ( $n$-degree of the polynomial used in approximation)) inside the integration range. Now, the value 


\section{Numerical evaluation of fractional differ-integrals...}

of a derivative (as well as the accuracy of numerical integration) does depend on the shape of the function! So, it is always the quest for the optimal shape of the integrand.

For the method GrLET and NCM we used L = 4, 15, 32, 61 and 600 intervals.

For GaLEG method: $\mathrm{L}=4,15$ and 32 intervals only.

For GaKRO we used following pairs: G7/K15 which is the industry standard in numerical integration [14] along with G30/K61. The pairs correspond to $\mathrm{L}=15$ and 61 intervals respectively.

It is widely known, that number of $\mathrm{L}$ greater than $38-46$ for the Gauss methods often causes the error rises rapidly. Sometimes $100 \%$ and more! That's why you will encounter empty fields in all tables with results for these methods.

\section{The IMT Transformation ( $m R L)$ explained}

The integral of RL differ-integral formulas (1), (3) is not only fast-changing but also includes improper integral which has singularity at end of the integration range (Fig. 2).

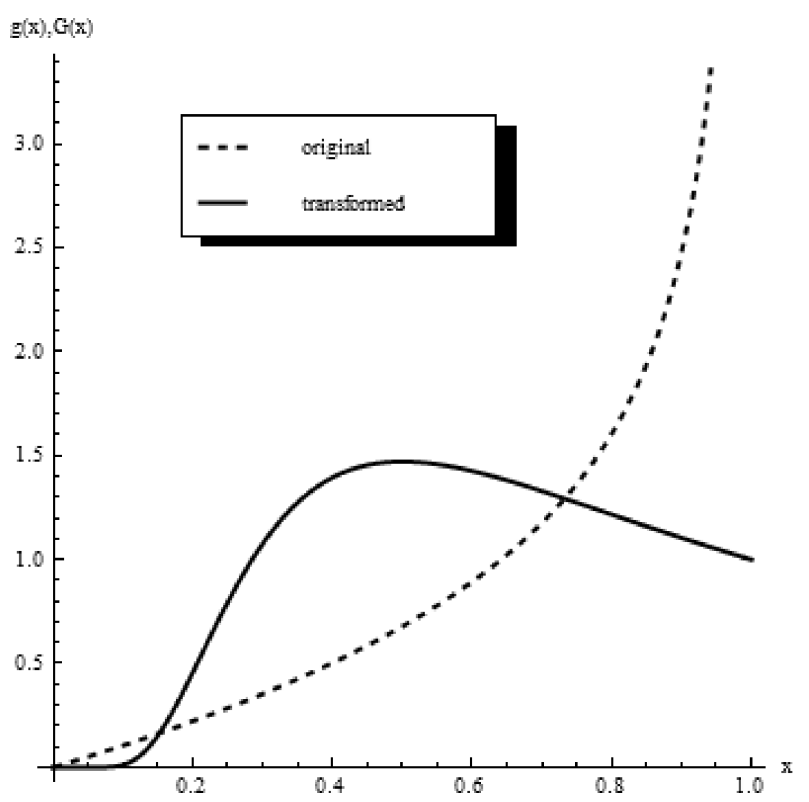

Fig. 2. Graphs of the original and modified integrand of the RL formula via (9) of the function $f(t)=\sin t$

This means, knowing the weaknesses of applied methods of numerical integration, that our goals were to transform the integrand of the RL formulas to remove the singularity and make it "smoother".

The IMT Transformation is a way of independent variable transformation proposed by three Japanese mathematicians: Iri, Moriguti and Takasawa in 1970 [16].

This transformation uses the basic idea standing behind the expression substituting used in analytic integration. But this time it lets to obtain a desired shape of the integrand instead of the simplest analytical formula of it.
The transformation can be applied to each function under the condition of transforming the calculated expression and possibly range of the integration via the substituting expression.

Below, there is presented the worked example of the above described technique for $f(t)=1$, and $t_{0}=0, t=1$, fractional integral of the order $\nu=0.5$.

The variable change $1-x \rightarrow e^{1-1 / u}$ (9) removes the singularity from the integrand. It also makes it "smoother". The transformation is visualized in Figs. 3-5.

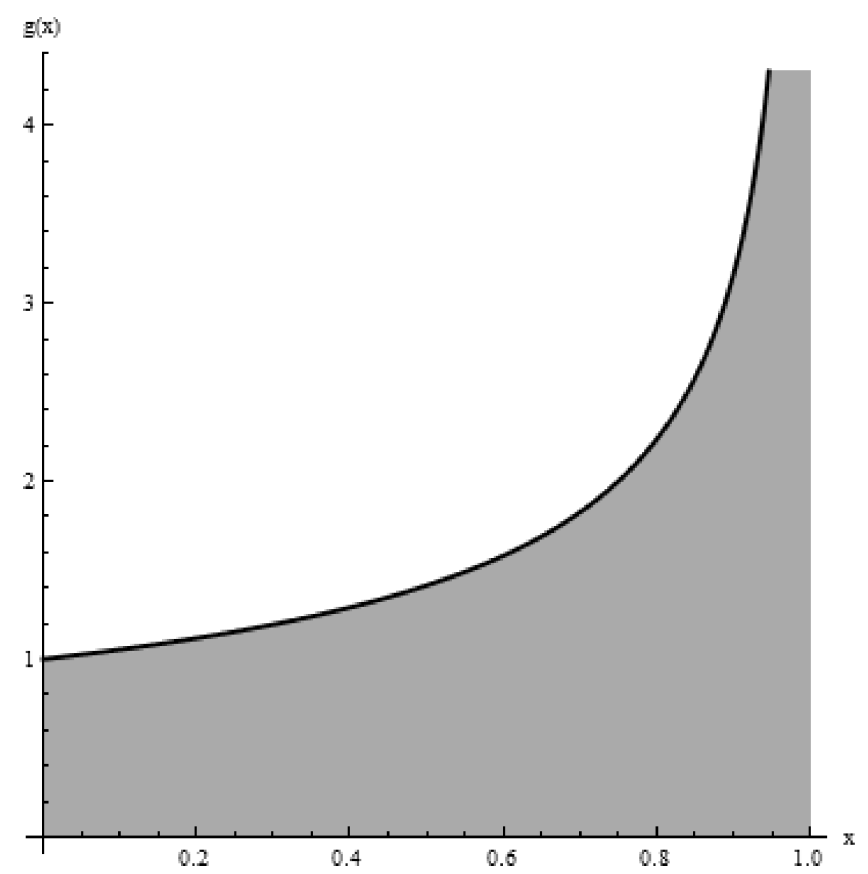

Fig. 3. Graph of the original integrand $g(x)$

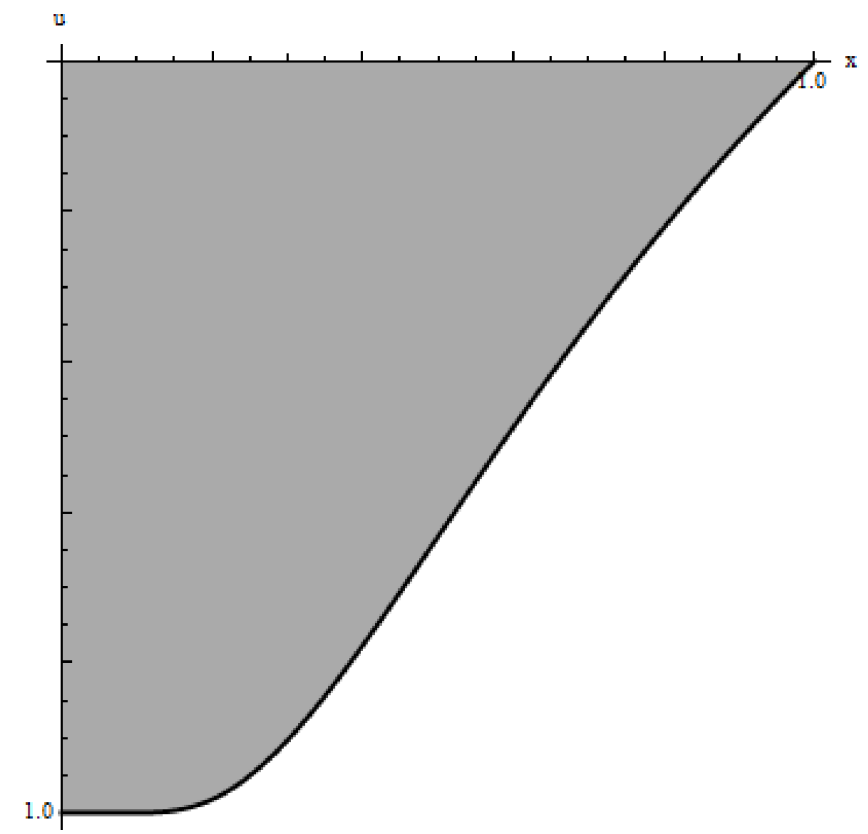

Fig. 4. Graph of the transformed variable $\mathrm{x}$ into $\mathrm{u}$ 


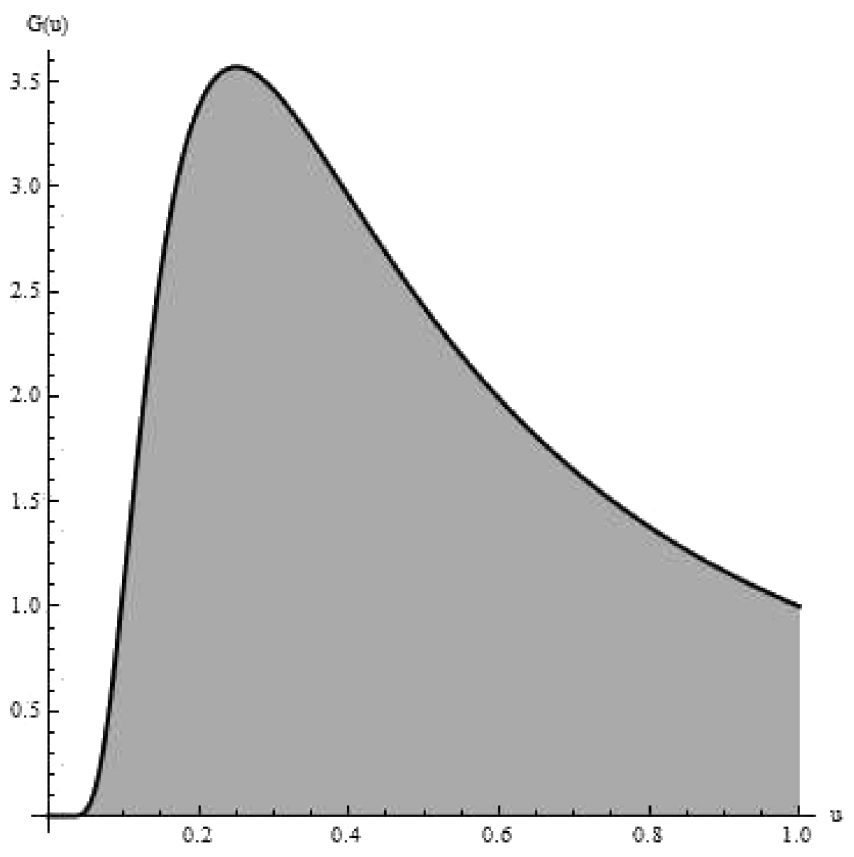

Fig. 5. Graph of the transformed integrand G(u)

The integration range has to be transformed using formula (11) as well.

$$
\begin{gathered}
\int_{0}^{1} \underbrace{(1-x)^{-0.5}}_{g(x)} d x=\left|\begin{array}{c}
1-x=e^{1-\frac{1}{u}} \\
-d x=e^{1-\frac{1}{u}} \cdot \frac{1}{u^{2}} d u \\
d x=\left(-e^{1-\frac{1}{u}}\right) \cdot \frac{1}{u^{2}} d u
\end{array}\right|=\ldots \\
\ldots=\int_{0}^{1} \underbrace{\left(e^{1-\frac{1}{u}}\right)^{0.5} \cdot \frac{1}{u^{2}}}_{G(u)} d u \\
x=1-e^{1-\frac{1}{u}}, \\
u=\frac{1}{1-\ln (1-x)} .
\end{gathered}
$$

Above technique is visualized in Figs. 3-5.

The original IMT Transformation goal was to transform the independent variable to make all derivatives of the new integrand vanish at the end of the integration range. Then, usually trapezoidal rule was applied. We use different approach we apply Newton-Cotes' Midpoint Rule and Gauss formulas.

Because in the original IMT Transformation an upper integration range may not exceed, in our case, the value of $\pi-e$, we had to modify the substitution accordingly. It was done in the arbitrary way.

\section{The test results}

First $t_{0} I_{t}^{\nu} f(t)$ for $f(t)=\sin (t), t \in(0 ; 2 \pi)$, the order $\nu=0.2,0.5,0.8$ using modified RL formula (1) via $\mathrm{mRL}$ was evaluated. The results are presented in Tables $2 \mathrm{a}-2 \mathrm{c}$.
Table 2a

Obtained values of absolute error for $\nu=0.2$

\begin{tabular}{ccccc}
\hline \hline $\mathrm{L}$ & GrLET & RL NCM & RL GaLEG & RL GaKRO \\
\hline 4 & $1.379 \mathrm{e}-01$ & $1.207 \mathrm{e}-02$ & $4.748 \mathrm{e}-03$ & - \\
\hline 15 & $3.873 \mathrm{e}-02$ & $3.111 \mathrm{e}-03$ & $2.333 \mathrm{e}-04$ & $1.352 \mathrm{e}-02$ \\
\hline 32 & $1.836 \mathrm{e}-02$ & $1.318 \mathrm{e}-03$ & $\mathbf{3 . 9 4 3 e - 0 5}$ & - \\
\hline 61 & $9.682 \mathrm{e}-03$ & $6.225 \mathrm{e}-04$ & - & $\mathbf{4 . 5 9 9 e - 0 6}$ \\
\hline 600 & $\mathbf{9 . 8 2 3 e - 0 4}$ & $\mathbf{4 . 1 2 6 e - 0 5}$ & - & - \\
\hline 4 & & $1.116 \mathrm{e}-01$ & $9.330 \mathrm{e}-02$ & - \\
\hline 15 & & $8.037 \mathrm{e}-03$ & $2.751 \mathrm{e}-09$ & $1.638 \mathrm{e}-08$ \\
\hline 32 & & $1.626 \mathrm{e}-03$ & $\mathbf{1 . 2 8 3 e - 1 1}$ & - \\
\hline 61 & & $4.401 \mathrm{e}-04$ & - & $\mathbf{2 . 7 7 6 e - 1 6}$ \\
\hline 600 & & $\mathbf{4 . 5 2 1 e - 0 6}$ & - & - \\
\hline $\mathrm{L}$ & GrLET & mRL NCM & mRL GaLEG & mRL GaKRO \\
\hline
\end{tabular}

Table $2 b$

Obtained values of absolute error for $\nu=0.5$

\begin{tabular}{ccccc}
\hline \hline $\mathrm{L}$ & GrLET & RL NCM & RL GaLEG & RL GaKRO \\
\hline 4 & $2.507 \mathrm{e}-01$ & $4.620 \mathrm{e}-02$ & $1.077 \mathrm{e}-02$ & - \\
\hline 15 & $7.047 \mathrm{e}-02$ & $7.691 \mathrm{e}-03$ & $2.462 \mathrm{e}-04$ & $1.196 \mathrm{e}-04$ \\
\hline 32 & $3.347 \mathrm{e}-02$ & $2.627 \mathrm{e}-03$ & $\mathbf{2 . 2 6 4 e - 0 5}$ & - \\
\hline 61 & $1.776 \mathrm{e}-02$ & $1.036 \mathrm{e}-03$ & - & $\mathbf{1 . 7 4 1 e - 0 6}$ \\
\hline 600 & $\mathbf{1 . 8 0 6 e - 0 3}$ & $\mathbf{3 . 5 7 8 e - 0 5}$ & - & - \\
\hline 4 & & $3.157 \mathrm{e}-01$ & $5.448 \mathrm{e}-01$ & - \\
\hline 15 & & $3.610 \mathrm{e}-02$ & $1.029 \mathrm{e}-08$ & $2.165 \mathrm{e}-07$ \\
\hline 32 & & $7.308 \mathrm{e}-03$ & $\mathbf{5 . 5 5 1 e - 1 2}$ & - \\
\hline 61 & & $1.987 \mathrm{e}-03$ & - & $\mathbf{4 . 9 9 6 e - 1 6}$ \\
\hline 600 & & $\mathbf{2 . 0 3 2 e - 0 5}$ & - & - \\
\hline $\mathrm{L}$ & GrLET & $\mathrm{mRL} \mathrm{NCM}$ & $\mathrm{mRL} \mathrm{GaLEG}$ & $\mathrm{mRL}$ GaKRO \\
\hline
\end{tabular}

Table 2c

Obtained values of absolute error for $\nu=0.8$

\begin{tabular}{ccccc}
\hline \hline $\mathrm{L}$ & GrLET & RL NCM & RL GaLEG & RL GaKRO \\
\hline 4 & $1.758 \mathrm{e}-01$ & $4.137 \mathrm{e}-02$ & $6.113 \mathrm{e}-03$ & - \\
\hline 15 & $4.799 \mathrm{e}-02$ & $4.984 \mathrm{e}-03$ & $6.108 \mathrm{e}-05$ & $2.410 \mathrm{e}-05$ \\
\hline 32 & $2.268 \mathrm{e}-02$ & $1.427 \mathrm{e}-03$ & $\mathbf{4 . 2 2 1 e - 0 6}$ & - \\
\hline 61 & $1.194 \mathrm{e}-02$ & $4.827 \mathrm{e}-04$ & - & $\mathbf{1 . 4 9 5 e - 0 7}$ \\
\hline 600 & $\mathbf{1 . 2 1 9 e - 0 3}$ & $\mathbf{9 . 4 5 5 e - 0 6}$ & - & - \\
\hline 4 & & $4.370 \mathrm{e}-01$ & $1.512 \mathrm{e}+00$ & - \\
\hline 15 & & $9.517 \mathrm{e}-02$ & $7.148 \mathrm{e}-08$ & $8.654 \mathrm{e}-07$ \\
\hline 32 & & $1.930 \mathrm{e}-02$ & $\mathbf{2 . 0 9 9 e - 1 1}$ & - \\
\hline 61 & & $5.227 \mathrm{e}-03$ & - & $\mathbf{1 . 2 2 1 e - 1 5}$ \\
\hline 600 & & $\mathbf{5 . 3 7 0 e - 0 5}$ & - & - \\
\hline $\mathrm{L}$ & GrLET & mRL NCM & mRL GaLEG & mRL GaKRO \\
\hline
\end{tabular}

Each table is arranged as follows: L denotes number of nodes used in approximation by every method; the upper part of the table contains the absolute errors values for all methods applied to unmodified integrands; the bottom part contains the absolute errors values for all methods applied to transformed integrands; the bolded values represent the best results (lowest values of the absolute errors) achieved by each method. 
Numerical evaluation of fractional differ-integrals...

For each tested function we present three tables. Each contains the values of the absolute error for different order of expression calculated.

${ }_{0} I_{2 \pi}^{\nu} f(t)=\frac{1}{\Gamma(\nu)} \int_{0}^{2 \pi}\left(e^{2 \pi-\frac{2 \pi}{t}}\right)^{\nu} \frac{2 \pi}{t^{2}} \sin \left(2 \pi-e^{2 \pi-\frac{2 \pi}{t}}\right) d t$.

In case of the function (7), as it is easy to notice, the only methods which benefit from the integrand transformation are Gaussian methods. The accuracy increased 2-3 times.

Next the same integrals are obtained for the function $f(t)=1.5 \cos (2 t)+2.2 \cos (4 t), t \in(0 ; 2 \pi), \quad \nu=$ $0.2,0.5,0.8$

Modified RL formula (1) via mRL assumes the following form

$$
\begin{aligned}
& { }_{0} I_{2 \pi}^{\nu} f(t)=\frac{1}{\Gamma(\nu)} \int_{0}^{2 \pi}\left(e^{2 \pi-\frac{2 \pi}{t}}\right)^{\nu} \frac{2 \pi}{t^{2}} \\
& \quad \times 1.5 \cos \left(2\left(2 \pi-e^{2 \pi-\frac{2 \pi}{t}}\right)\right) \\
& +2.2 \cos \left(4\left(2 \pi-e^{2 \pi-\frac{2 \pi}{t}}\right)\right) d t .
\end{aligned}
$$

The results are presented as plots in Tables 3a-3c.

Table 3a

Obtained values of absolute error for $\nu=0.2$

\begin{tabular}{ccccc}
\hline \hline $\mathrm{L}$ & GrLET & RL NCM & RL GaLEG & RL GaKRO \\
\hline 4 & $1.932 \mathrm{e}+00$ & $4.556 \mathrm{e}+00$ & $3.201 \mathrm{e}+00$ & - \\
\hline 15 & $1.017 \mathrm{e}-01$ & $2.245 \mathrm{e}+00$ & $1.495 \mathrm{e}+00$ & $1.379 \mathrm{e}+00$ \\
\hline 32 & $4.224 \mathrm{e}-02$ & $1.915 \mathrm{e}+00$ & $\mathbf{1 . 1 1 2 e + 0 0}$ & - \\
\hline 61 & $2.110 \mathrm{e}-02$ & $1.683 \mathrm{e}+00$ & - & $\mathbf{7 . 8 6 0 e - 0 1}$ \\
\hline 600 & $\mathbf{2 . 0 4 5 e - 0 3}$ & $\mathbf{1 . 0 6 5 e + 0 0}$ & - & - \\
\hline 4 & & $9.428 \mathrm{e}-01$ & $1.444 \mathrm{e}+00$ & - \\
\hline 15 & & $4.369 \mathrm{e}-01$ & $1.663 \mathrm{e}-01$ & $1.642 \mathrm{e}-01$ \\
\hline 32 & & $6.424 \mathrm{e}-04$ & $\mathbf{3 . 1 2 0 e}-10$ & - \\
\hline 61 & & $6.138 \mathrm{e}-05$ & - & $\mathbf{6 . 7 5 0 e - 1 4}$ \\
\hline 600 & & $\mathbf{6 . 6 3 6 e - 0 7}$ & - & - \\
\hline $\mathrm{L}$ & GrLET & mRL NCM & mRL GaLEG & mRL GaKRO \\
\hline
\end{tabular}

Table $3 b$

Obtained values of absolute error for $\nu=0.5$

\begin{tabular}{ccccc}
\hline \hline $\mathrm{L}$ & GrLET & RL NCM & RL GaLEG & RL GaKRO \\
\hline 4 & $5.570 \mathrm{e}+00$ & $6.804 \mathrm{e}+00$ & $3.204 \mathrm{e}+00$ & - \\
\hline 15 & $2.915 \mathrm{e}-01$ & $8.369 \mathrm{e}-01$ & $2.939 \mathrm{e}-01$ & $2.390 \mathrm{e}-01$ \\
\hline 32 & $1.397 \mathrm{e}-01$ & $5.627 \mathrm{e}-01$ & $\mathbf{1 . 4 0 2 e - 0 1}$ & - \\
\hline 61 & $7.439 \mathrm{e}-02$ & $4.059 \mathrm{e}-01$ & - & $\mathbf{5 . 8 5 4 e - 0 2}$ \\
\hline 600 & $\mathbf{7 . 7 0 0 e - 0 3}$ & $\mathbf{1 . 2 9 2 e - 0 1}$ & - & - \\
\hline 4 & & $4.602 \mathrm{e}+00$ & $3.418 \mathrm{e}+00$ & - \\
\hline 15 & & $1.800 \mathrm{e}+00$ & $6.337 \mathrm{e}-01$ & $7.296 \mathrm{e}-01$ \\
\hline 32 & & $5.812 \mathrm{e}-03$ & $\mathbf{2 . 9 9 9 e - 0 8}$ & - \\
\hline 61 & & $2.428 \mathrm{e}-04$ & - & $\mathbf{5 . 8 4 3 e - 0 8}$ \\
\hline 600 & & $\mathbf{5 . 5 6 2 e - 0 7}$ & - & - \\
\hline L & GrLET & mRL NCM & mRL GaLEG & mRL GaKRO \\
\hline
\end{tabular}

Table 3c

Obtained values of absolute error for $\nu=0.8$

\begin{tabular}{ccccc}
\hline \hline $\mathrm{L}$ & GrLET & RL NCM & RL GaLEG & RL GaKRO \\
\hline 4 & $1.013 \mathrm{e}+00$ & $1.047 \mathrm{e}+00$ & $4.396 \mathrm{e}+00$ & - \\
\hline 15 & $2.488 \mathrm{e}-01$ & $1.811 \mathrm{e}-01$ & $3.184 \mathrm{e}-02$ & $2.262 \mathrm{e}-02$ \\
\hline 32 & $1.225 \mathrm{e}-01$ & $9.520 \mathrm{e}-02$ & $\mathbf{9 . 7 4 5 e - 0 3}$ & - \\
\hline 61 & $6.594 \mathrm{e}-02$ & $5.642 \mathrm{e}-02$ & - & $\mathbf{2 . 3 8 0 e - 0 3}$ \\
\hline 600 & $\mathbf{1 . 2 6 7 e - 0 1}$ & $\mathbf{9 . 0 3 9 e - 0 3}$ & - & - \\
\hline 4 & & $1.034 \mathrm{e}+00$ & $3.845 \mathrm{e}+00$ & - \\
\hline 15 & & $4.361 \mathrm{e}-01$ & $1.376 \mathrm{e}+00$ & $1.806 \mathrm{e}+00$ \\
\hline 32 & & $2.307 \mathrm{e}-02$ & $\mathbf{2 . 4 4 4 e - 0 7}$ & - \\
\hline 61 & & $1.775 \mathrm{e}-03$ & - & $\mathbf{2 . 6 6 5 e - 1 5}$ \\
\hline 600 & & $\mathbf{1 . 1 1 3 e - 0 5}$ & - & - \\
\hline L & GrLET & mRL NCM & mRL GaLEG & mRL GaKRO \\
\hline
\end{tabular}

In case of the function (8), all of three methods benefited from the transformed integrand, although in a different way. There is 2-5 times accuracy increase to notice.

Now a problem of the fractional derivative ${ }_{t_{0}} D_{t}^{\nu} f(t)$ of the function $f(t)=\sin (t), t \in(0 ; 2 \pi), \nu=0.2,0.5,0.8$ is considered. We calculate

$$
n=\lceil\nu\rceil+1
$$

The modified RL formula (3) via mRL assumes the form

$$
\begin{gathered}
{ }_{0} D_{2 \pi}^{\nu} f(t)=\frac{(2 \pi)^{-\nu} \sin (0)}{\Gamma(1-\nu)} \\
\times+\frac{1}{\Gamma(n-\nu)} \int_{0}^{2 \pi}\left(e^{2 \pi-\frac{2 \pi}{t}}\right)^{n-\nu} \\
\cdot \frac{2 \pi}{t^{2}} \cos \left(2 \pi-e^{2 \pi-\frac{2 \pi}{t}}\right) d t .
\end{gathered}
$$

The obtained results are presented in Tables $4 \mathrm{a}-4 \mathrm{c}$.

Table $4 \mathrm{a}$

Obtained values of absolute error for $\nu=0.2$

\begin{tabular}{ccccc}
\hline \hline $\mathrm{L}$ & GrLET & RL NCM & RL GaLEG & RL GaKRO \\
\hline 4 & $1.522 \mathrm{e}-01$ & $1.408 \mathrm{e}-01$ & $5.803 \mathrm{e}-02$ & - \\
\hline 15 & $4.035 \mathrm{e}-02$ & $4.679 \mathrm{e}-02$ & $8.614 \mathrm{e}-03$ & $6.114 \mathrm{e}-03$ \\
\hline 32 & $1883 \mathrm{e}-02$ & $2.548 \mathrm{e}-02$ & $\mathbf{2 . 6 3 4 e - 0 3}$ & - \\
\hline 61 & $9.855 \mathrm{e}-03$ & $1.521 \mathrm{e}-02$ & - & $\mathbf{6 . 4 3 1 e - 0 4}$ \\
\hline 600 & $\mathbf{9 . 9 9 5 e - 0 4}$ & $\mathbf{2 . 4 4 3 e - 0 3}$ & - & - \\
\hline 4 & & $2.278 \mathrm{e}+00$ & $8.263 \mathrm{e}-01$ & - \\
\hline 15 & & $9.612 \mathrm{e}-03$ & $1.848 \mathrm{e}-07$ & $5.664 \mathrm{e}-07$ \\
\hline 32 & & $1.242 \mathrm{e}-03$ & $\mathbf{3 . 2 2 3 e - 0 7}$ & - \\
\hline 61 & & $3.033 \mathrm{e}-04$ & - & $\mathbf{3 . 5 0 1 e - 1 6}$ \\
\hline 600 & & $\mathbf{2 . 9 9 4 e - 0 4}$ & - & - \\
\hline $\mathrm{L}$ & GrLET & mRL NCM & mRL GaLEG & mRL GaKRO \\
\hline
\end{tabular}


Table $4 b$

Obtained values of absolute error for $\nu=0.5$

\begin{tabular}{ccccc}
\hline \hline L & GrLET & RL NCM & RL GaLEG & RL GaKRO \\
\hline 4 & $3.414 \mathrm{e}-01$ & $4.406 \mathrm{e}-01$ & $2.745 \mathrm{e}-01$ & - \\
\hline 15 & $8.021 \mathrm{e}-02$ & $2.213 \mathrm{e}-01$ & $7.944 \mathrm{e}-02$ & $4.640 \mathrm{e}-02$ \\
\hline 32 & $3.621 \mathrm{e}-02$ & $1.513 \mathrm{e}-01$ & $\mathbf{3 . 7 9 8 e - 0 2}$ & - \\
\hline 61 & $1.867 \mathrm{e}-02$ & $1.095 \mathrm{e}-01$ & - & $\mathbf{1 . 5 8 2 e - 0 2}$ \\
\hline 600 & $\mathbf{3 . 4 9 2 e - 0 3}$ & $\mathbf{3 . 5 7 8 e - 0 5}$ & - & - \\
\hline 4 & & $9.224 \mathrm{e}-01$ & $4.658 \mathrm{e}-01$ & - \\
\hline 15 & & $1.535 \mathrm{e}-03$ & $4.475 \mathrm{e}-07$ & $1.173 \mathrm{e}-06$ \\
\hline 32 & & $1.054 \mathrm{e}-04$ & $\mathbf{1 . 9 8 5 e - 0 8}$ & - \\
\hline 61 & & $1.916 \mathrm{e}-05$ & - & $\mathbf{1 . 8 2 3 e - 0 8}$ \\
\hline 600 & & $\mathbf{1 . 4 4 1 e - 0 7}$ & - & - \\
\hline L & GrLET & mRL NCM & mRL GaLEG & mRL GaKRO \\
\hline
\end{tabular}

Table $4 \mathrm{c}$

Obtained values of absolute error for $\nu=0.8$

\begin{tabular}{ccccc}
\hline \hline $\mathrm{L}$ & GrLET & RL NCM & RL GaLEG & RL GaKRO \\
\hline 4 & $4.101 \mathrm{e}-01$ & $7.928 \mathrm{e}-01$ & $6.619 \mathrm{e}-01$ & - \\
\hline 15 & $7.020 \mathrm{e}-02$ & $6.024 \mathrm{e}-01$ & $4.040 \mathrm{e}-01$ & $3.780 \mathrm{e}-01$ \\
\hline 32 & $2.846 \mathrm{e}-02$ & $5.174 \mathrm{e}-01$ & $\mathbf{3 . 0 0 5 e - 0 1}$ & - \\
\hline 61 & $1.382 \mathrm{e}-02$ & $4.547 \mathrm{e}-01$ & - & $\mathbf{2 . 1 2 4 e - 0 1}$ \\
\hline 600 & $\mathbf{1 . 3 1 2 e - 0 3}$ & $\mathbf{2 . 8 7 8 e - 0 1}$ & - & - \\
\hline 4 & & $2.553 \mathrm{e}-01$ & $1.907 \mathrm{e}-01$ & - \\
\hline 15 & & $2.762 \mathrm{e}-05$ & $2.003 \mathrm{e}-05$ & $3.204 \mathrm{e}-07$ \\
\hline 32 & & $5.749 \mathrm{e}-05$ & $\mathbf{1 . 6 1 2 e - 0 7}$ & - \\
\hline 61 & & $1.714 \mathrm{e}-05$ & - & $\mathbf{1 . 3 7 4 e - 0 7}$ \\
\hline 600 & & $\mathbf{3 . 1 7 2 e - 0 7}$ & - & - \\
\hline $\mathrm{L}$ & GrLET & mRL NCM & mRL GaLEG & mRL GaKRO \\
\hline
\end{tabular}

In case of the function (7) and calculated fractional derivative, all of three methods benefited in similar way from the integrand transformation. There is maximum 7-times accuracy increase to notice.

Finally we calculate $t_{0} D_{t}^{\nu} f(t)$, of the function $f(t)=$ $1.5 \cos (2 t)+2.2 \cos (4 t), t \in(0 ; 2 \pi), \nu=0.2,0.5,0.8$. Under the condition (14) modified RL formula (3) via mRL assumes the form

$$
\begin{aligned}
{ }_{0} D_{2 \pi}^{\nu} f(t) & =+\frac{1}{\Gamma(n-\nu)} \int_{0}^{2 \pi}\left(e^{2 \pi-\frac{2 \pi}{t}}\right)^{n-\nu} \cdot \frac{2 \pi}{t^{2}} \\
\times & \left(-3 \sin \left(2\left(2 \pi-e^{2 \pi-\frac{2 \pi}{t}}\right)\right)\right. \\
- & \left.8.8 \sin \left(4\left(2 \pi-e^{2 \pi-\frac{2 \pi}{t}}\right)\right)\right) d t .
\end{aligned}
$$

Obtained results are presented in Tables 5a-5c.

In case of the function (8), and fractional derivative, using the method GaKRO applied to transformed integrand allowed 3-5 accuracy increase. The other methods increased the accuracy in a minimal way.
Table 5a

Obtained values of absolute error for $\nu=0.2$

\begin{tabular}{ccccc}
\hline \hline $\mathrm{L}$ & GrLET & RL NCM & RL GaLEG & RL GaKRO \\
\hline 4 & $1.450 \mathrm{e}+01$ & $1.162 \mathrm{e}+00$ & $3.809 \mathrm{e}+00$ & - \\
\hline 15 & $8.693 \mathrm{e}-02$ & $2.161 \mathrm{e}-01$ & $2.506 \mathrm{e}-03$ & $2.262 \mathrm{e}-02$ \\
\hline 32 & $7.197 \mathrm{e}-02$ & $5.947 \mathrm{e}-02$ & $\mathbf{1 . 7 4 0 e - 0 4}$ & - \\
\hline 61 & $4.432 \mathrm{e}-02$ & $1.995 \mathrm{e}-02$ & - & $\mathbf{2 . 3 8 0 e - 0 3}$ \\
\hline 600 & $\mathbf{5 . 1 6 6 e - 0 3}$ & $\mathbf{3 . 8 9 5 e - 0 4}$ & - & - \\
\hline 4 & & $3.482 \mathrm{e}+01$ & $1.376 \mathrm{e}+00$ & - \\
\hline 15 & & $2.624 \mathrm{e}+00$ & $1.950 \mathrm{e}+00$ & $3.512 \mathrm{e}+00$ \\
\hline 32 & & $1.128 \mathrm{e}+00$ & $\mathbf{1 . 3 6 6 e - 0 5}$ & - \\
\hline 61 & & $2.344 \mathrm{e}-01$ & - & $\mathbf{3 . 5 3 3 e - 1 5}$ \\
\hline 600 & & $\mathbf{2 . 2 1 4 e - 0 3}$ & - & - \\
\hline $\mathrm{L}$ & GrLET & mRL NCM & mRL GaLEG & mRL GaKRO \\
\hline
\end{tabular}

Table $5 b$

Obtained values of absolute error for $\nu=0.5$

\begin{tabular}{ccccc}
\hline \hline $\mathrm{L}$ & GrLET & RL NCM & RL GaLEG & RL GaKRO \\
\hline 4 & $2.344 \mathrm{e}+00$ & $2.305 \mathrm{e}+00$ & $4.958 \mathrm{e}+00$ & - \\
\hline 15 & $1.025 \mathrm{e}+00$ & $3.301 \mathrm{e}-01$ & $1.012 \mathrm{e}-02$ & $4.931 \mathrm{e}-03$ \\
\hline 32 & $6.415 \mathrm{e}-01$ & $1.093-01$ & $\mathbf{1 . 0 9 8 e - 0 3}$ & - \\
\hline 61 & $3.686 \mathrm{e}-02$ & $4.281 \mathrm{e}-02$ & - & $\mathbf{7 . 1 6 4 e - 0 5}$ \\
\hline 600 & $\mathbf{4 . 0 4 6 e - 0 2}$ & $\mathbf{1 . 4 7 4 e - 0 3}$ & - & - \\
\hline 4 & & $1.851 \mathrm{e}-01$ & $4.834 \mathrm{e}-01$ & - \\
\hline 15 & & $7.531 \mathrm{e}-01$ & $4.154 \mathrm{e}-01$ & $2.052 \mathrm{e}+00$ \\
\hline 32 & & $4.276 \mathrm{e}-01$ & $\mathbf{5 . 5 9 7 e - 0 6}$ & - \\
\hline 61 & & $8.873 \mathrm{e}-02$ & - & $\mathbf{1 . 9 4 5 e - 1 3}$ \\
\hline 600 & & $\mathbf{8 . 3 8 1 e - 0 4}$ & - & - \\
\hline $\mathrm{L}$ & GrLET & mRL NCM & mRL GaLEG & mRL GaKRO \\
\hline
\end{tabular}

Table 5c

Obtained values of absolute error for $\nu=0.8$

\begin{tabular}{ccccc}
\hline \hline $\mathrm{L}$ & GrLET & RL NCM & RL GaLEG & RL GaKRO \\
\hline 4 & $9.027 \mathrm{e}+00$ & $1.874 \mathrm{e}+00$ & $2.334 \mathrm{e}+00$ & - \\
\hline 15 & $3.727 \mathrm{e}+00$ & $1.320 \mathrm{e}-01$ & $9.600 \mathrm{e}-03$ & $5.570 \mathrm{e}-03$ \\
\hline 32 & $2.154 \mathrm{e}+00$ & $5.486 \mathrm{e}-02$ & $\mathbf{1 . 6 2 4 e - 0 3}$ & - \\
\hline 61 & $1.197 \mathrm{e}+00$ & $2.570 \mathrm{e}-02$ & - & $\mathbf{1 . 8 9 4 e - 0 4}$ \\
\hline 600 & $\mathbf{1 . 2 6 7 e - 0 1}$ & $\mathbf{1 . 7 0 0 e - 0 3}$ & - & - \\
\hline 4 & & $6.027 \mathrm{e}+00$ & $2.323 \mathrm{e}-01$ & - \\
\hline 15 & & $9.484 \mathrm{e}-02$ & $1.587 \mathrm{e}-02$ & $6.566 \mathrm{e}-01$ \\
\hline 32 & & $9.527 \mathrm{e}-02$ & $\mathbf{1 . 3 4 3 e - 0 6}$ & - \\
\hline 61 & & $1.974 \mathrm{e}-02$ & - & $\mathbf{1 . 2 4 3 e - 1 4}$ \\
\hline 600 & & $\mathbf{1 . 8 6 4 e - 0 4}$ & - & - \\
\hline $\mathrm{L}$ & GrLET & mRL NCM & mRL GaLEG & mRL GaKRO \\
\hline
\end{tabular}

\section{Final conclusions}

1. The point of reference in this experiment was the method GrLET (4) and the value $10^{-4}$, which is possible to achieve using 600 of coefficients (5) (equivalent to sample points). In our previous paper [17] we discovered that the shape of the integrand does not influence accuracy of the calculations applying GrLET method to non-periodic functions. The most influential in this context were: number 


\section{Numerical evaluation of fractional differ-integrals...}

of coefficients applied and accuracy of their calculation. As stated above, applying maximum number of 600 of coefficients (such amount is often used in practical, technical applications) we were able to obtain values with maximum the accuracy $10^{-4}$. The accuracy of the calculations increased linearly. In case of the periodic functions presented in this paper, the level of accuracy increased proportionally as well. However, it was much lower. Satisfactory level of accuracy $10^{-4}$ cannot be reach applying GrLET with less than 600 of the coefficients (see the column GrLET in Tables $2 \mathrm{a}-5 \mathrm{c}$ ).

2. The shape of integrand influences the accuracy of the calculations when applying methods of numerical integration using RL and mRL formulas (see the columns RL and $\mathrm{mRL}$ for all methods in tables $2 \mathrm{a}-05 \mathrm{c}$ ). The relationship between the shape of the integrand and the order of the derivative calculated applying RL formula (3) is presented in Fig. 6.

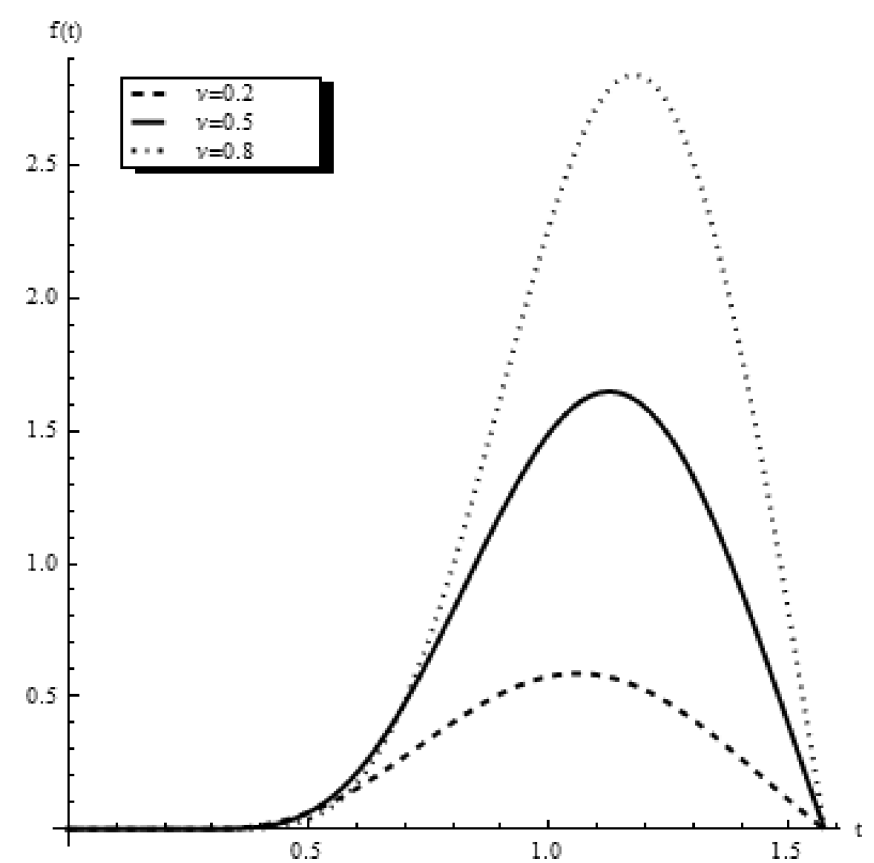

Fig. 6. Graphs of the modified integrand (12) of the RL formula

(3 in relation to $\nu(\mathrm{mRL})$

3. The values of the integrand obtained using unmodified RL formula are burdened with great absolute error (see column RL for all methods in tables $2 \mathrm{a} 0-5 \mathrm{c}$ ). This makes the formula often unsuitable in practical, technical applications.

4. The level of errors appeared because of the fact that the "core" integrand of the formula has "fast-changing" character and also has singularity at the end point of the integration range (see Fig. 2).

5. Applying the IMT Transformation with special substitute expression to the non-periodic integrands to "remove" the singularity and "smooth" it allowed not only to obtain way better results, especially with Gauss methods, than GrLET, but often using radical reduced number of sampling points (see columns mRL for all methods in Tables $2 \mathrm{a}-5 \mathrm{c}$ ). This lowers the level of calculation complexity in context of de- creasing amount of sample points. In case of periodic functions the IMT Transformation allowed not only to remove the singularity from the integrands but also caused them to become highly oscillated. This made the test difficult for applied methods of numerical integration. Especially for Newton Cotes' Midpoint Rule. It was possible to obtain way better results but often with maximum sample points anticipated for the test only (see Table 3c, 5a).

6. The values of absolute errors decreased proportionally to the order of differ-integrals (the values of $\nu$ ), when fractional integrals calculated and increased when fractional derivatives calculated. It is because the shape of the integrand in mRL formula becomes "unfriendly" for numerical integration while type and order of differ-integral calculated changes (notice Fig. 6).

7. The NCM is an universal tool. It does not depend so strongly as the GaLEG, on a shape and monotonicity of the integrand. Therefore it can be applied to any kind of integrands, also to these which have singularities [17]. In case of periodic functions, because of the oscillatory character of their mRL formula, in some cases accuracy of the calculations were only slightly better than unmodified RL formula (Table 4a, 4b)

8. Using GaLEG, when applied to the IMT transformed integrand, seems to be the only way, not only because of the low values of absolute error, but also because of the fact, that these low values are obtained with only 5-10\% sample points used by GrLET method and NCM. This can dramatically reduce the complexity of the calculations and their speed.

9. The variant of the IMT Transformation which was introduced in this paper can be applied to each function under the condition of transforming the calculated expression and possibly the range of the integration accordingly (1011). This makes the transformation complicated for function with other integration limits than $(0,1)$.

10. The IMT Transformation is an excellent choice for nonperiodic functions. This was proved in [17]. Applying this transformation to integrands with periodic character did allow to obtain better results $(1-15 \mathrm{x}$, see Tables $2 \mathrm{a}-5 \mathrm{c})$ but with maximum sample points planned in the test only.

11. The IMT Transformation in connection with Gauss methods make one excellent tool to solve problems of the accuracy of the differ-integrals of non-integer orders calculations for wide range type of functions.

12. The logic of our tools needed only the grade of the desired polynomial as an input data. The rest of data were calculated "on the fly" (the polynomial itself, its derivative, the abscissas and the weights). In practical applications we can and should use tabulated values (of abscissas and weights) which were the subject of standardization all over the world. This can yet reduce the complexity of calculations which then can make the method become perfect suitable in practical applications.

13. Above there are presented graphs of the calculated FOI/FOD (Figs. 7 and 8) of the function (7) and of the function (8) (Figs. 9 and 10). 
D. Brzeziński and P. Ostalczyk

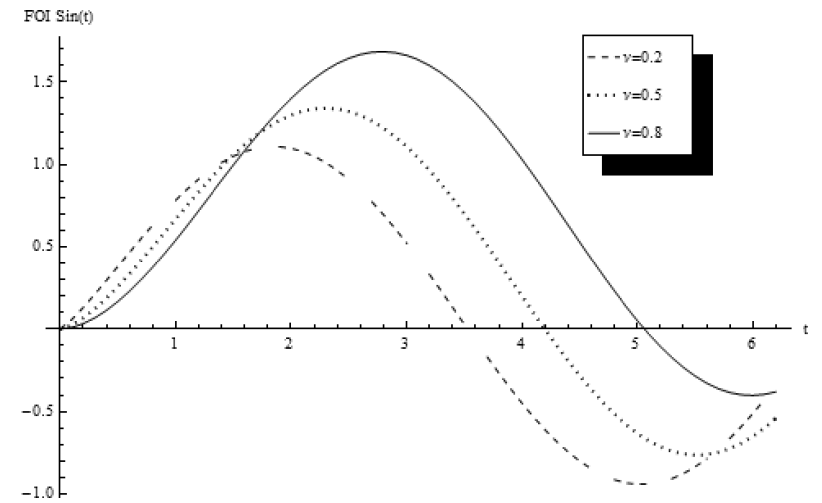

Fig. 7. Graph of the calculated FOI of the function (7)

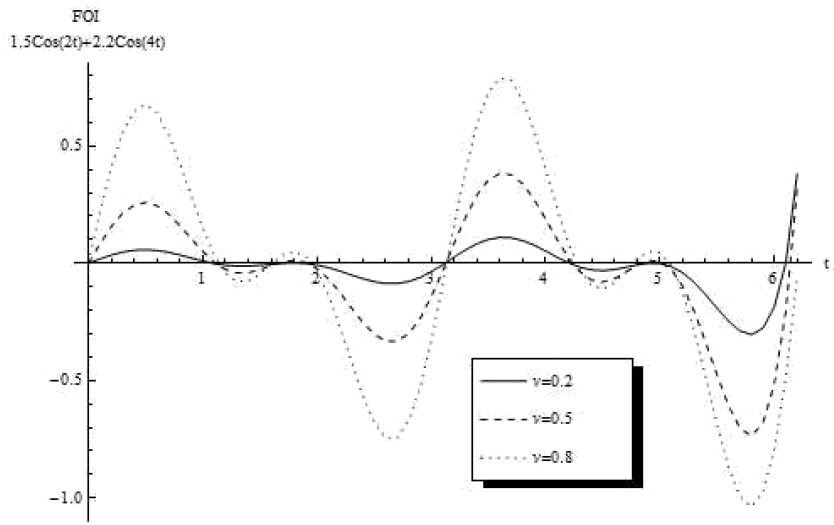

Fig. 9. Graph of the calculated FOI of the function (8)

\section{REFERENCES}

[1] A.Oustaloup, O. Cois, and L. Lelay, Représentation et Identification par Modèle non Entire, Hermes, Paris, 2005.

[2] J. Sabatier, O.P. Agrawal, and T.J.A. Machado, Advances in Fractional Calculus. Theoretical Developments and Applications in Physics and Engineering, Springer, Berlin, 2007.

[3] Y.Q. Chen, B.M. Vinagre, and I. Podlubny, "Continued fraction expansion approaches to discretizing fractional order derivatives - an expository review", Nonlinear Dynamics 38, 155170 (2004).

[4] P. Ostalczyk, "The non-integer difference of the discrete-time function and its application to the control system synthesis", Int. J. System Science 31 (12), 1551-1561 (2000).

[5] P. Ostalczyk, "Stability analysis of a discrete-time system with a variable-, fractional-order controller", Bull. Pol. Ac.: Tech. 58 (4), 613-619 (2010).

[6] M.Buslowicz, "Robust stability of positive discrete-time linear systems of fractional order", Bull. Pol. Ac.: Tech. 58 (4), 567-572 (2010).

[7] K. Diethelm, "An algorithm for the numerical solution of differential equations of fractional order", Electronic Trans. on Numerical Analysis 5, 1-6 (1977).

[8] C.H. Lubich, "Discretized fractional calculus", SIAM J.Mathematical Analysis 17, 704-719 (1986).

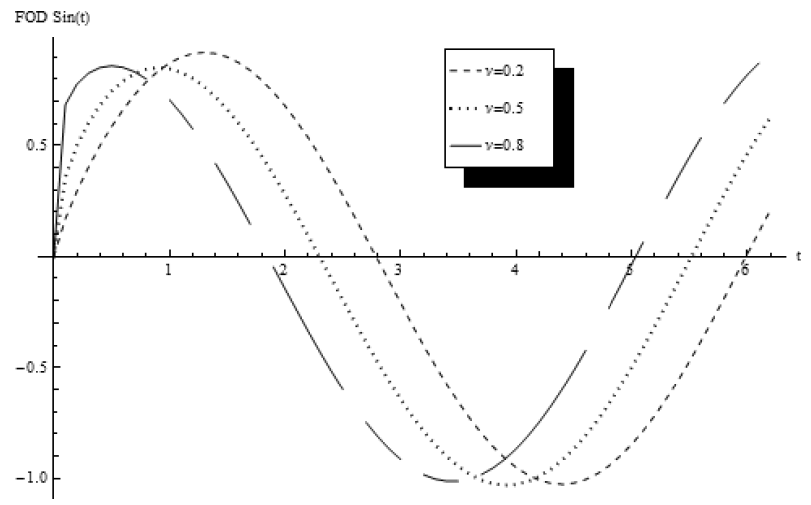

Fig. 8. Graph of the calculated FOD of the function (7)

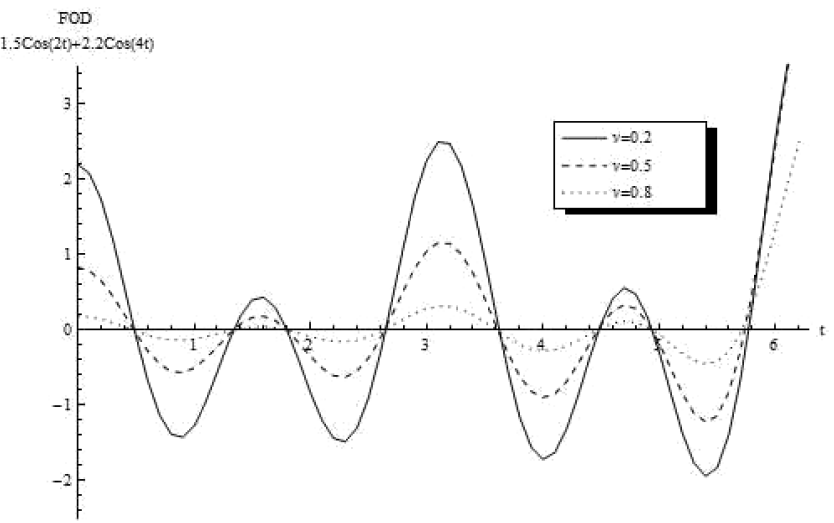

Fig. 10. Graph of the calculated FOD of the function (8)

[9] P. Ostalczyk, "The time-varying fractional order difference equations", Proc. Design Eng. Technical Conf. \& Computers and Information 1, 1-9 (1986).

[10] S. Samko, A. Kilbas, and O. Marichev, Fractional Integrals and Derivatives: Theory and Applications, Gordon and Breach, London, 1993.

[11] R.L. Burden and J.D. Faires, Numerical Analysis, Brooks/Cole Cengage Learning, Boston, 2003.

[12] R.A. Krommer and Ch.W. Ueberhuber, Computational Integration, SIAM, Philadelphia, 1986.

[13] A.H. Stroud and D. Secrest, Gaussian Quadrature Formulas, Prentice-Hall, Englewood Cliffs, 1966.

[14] D. Kahaner, C. Moler, and S. Nash, Numerical Methods and Software, Prentice Hall, New Jersey, 1989.

[15] P.K. Kythe and M.R. Schäferkotter, Handbook of Computational Methods for Integration, Chapman \& Hall/CRC, Ohio, 2005.

[16] H. Takahashi and M. Mori, "Quadrature formulas obtained by variable transformation", Numer. Math. 21, 201-216 (1973).

[17] D. Brzeziński and P. Ostalczyk, "Numerical evaluation of fractional differ-integral of some elementary functions via IMT transformation", Proc. 14th Int. Conf. on System Modelling and Control, SMC 1, CD-ROM (2011). 